\title{
Testing method and distribution characteristics of spatial pesticide spraying deposition quality balance for unmanned aerial vehicle
}

\author{
Changling Wang ${ }^{1}$, Xiongkui He ${ }^{1 *}$, Xiaonan Wang ${ }^{1}$, Zhichong Wang ${ }^{1}$, Shilin Wang ${ }^{1}$, \\ Longlong $\mathrm{Li}^{1}$, Jane Bonds ${ }^{2}$, Andreas Herbst ${ }^{3}$, Zhiguo Wang ${ }^{4}$ \\ (1. College of Science, China Agricultural University, Beijing 100193, China; 2. Bonds Consulting Group LLC, \\ Panama City Florida 32408, USA; 3. Institute for Application Techniques in Plant Protection, Julius Kühn-Institute (JKI), \\ Federal Research Centre for Cultivated Plants, Messeweg 11/12 D-38104 Braunschweig, Germany; \\ 4. Quanfeng Aviation Plant Protection Technology Co., Ltd., Anyang 455001, China)
}

\begin{abstract}
In order to explore the spatial pesticide spraying deposition distribution, the downwash flow field characteristics for unmanned aerial vehicle (UAV) pesticide application with accurate flight height and velocity and the relationship of these two aspects, a novel measurement method was proposed in this paper. A model ' $3 \mathrm{WQF} 80-10$ ' single-rotor diesel UAV was tested using this method in wheat field and the effects of flight direction, flight parameters and crosswind on the distribution of spatial spraying deposition quality balance (SSDQB) and the downwash flow field distribution were researched. A cuboid aluminum sampling frame of spatial spraying deposition quality balance (SFSSDQB) with monofilament wires was made for collecting the droplets in four directions, and a set of multi-channel micro-meteorology measurement system (MMMS) was applied for measuring the downwash wind speed in three directions. Besides, BeiDou Navigation Satellite System (BNSS) was used for controlling and recording the working height, velocity and track of this model of single-rotor UAV. The results showed the distribution of the spatial spray deposition and the downwash flow field of UAV could be measured effectively at exact flight height and velocity via this method. When the average wind speed was $0.9 \mathrm{~m} / \mathrm{s}$, the average temperature was $31.5^{\circ} \mathrm{C}$ and the average relative humidity was $34.1 \%$, and the average distribution ratios of spraying deposition for model ' 3 WQF $80-10$ ' UAV on the upwind part, the top part, the downwind part and the bottom part were $4.4 \%, 2.3 \%, 50.4 \%$ and $43.7 \%$, respectively. The flight directions of forward and backward had an impact on droplet deposition distribution and the working effect of flying backwards, with $60 \%$ of deposition ratio of the bottom part of the SFSSDQB, was better than flying forward. There was a linear negative correlation between the coefficient of variation $(\mathrm{CV})$ of the bottom part and the flight height and the coefficient of determination was 0.9178 , which means that the deposition distribution becomes more uniform with the increase of height. Additionally, there was a linear positive correlation between weighted mean deposition rate and crosswind speed and the coefficient of determination was 0.9684 , which shows the deposition distribution gets more concentrated towards the downwind part with the rise of crosswind speed. Therefore, according to the results of tests of downwash airflow speed, it is shown that regardless of the flight direction and height and the crosswind, all these factors influence the droplet deposition distribution via weakening the intensity of the downwash airflow field in the direction perpendicular to the ground. The results can provide valuable information for the research of UAV pesticide application techniques and the establishment of the standard of spraying deposition and drift tests of UAV in crop field.
\end{abstract}

Keywords: unmanned aerial vehicle (UAV), spatial spraying deposition, distribution, downwind airflow, flow field DOI: $10.25165 / \mathrm{j}$.jjabe. 20181102.3187

Citation: Wang C L, He X K, Wang X N, Wang Z C, Wang S L, Li L L, et al. Testing method and distribution characteristics of spatial pesticide spraying deposition quality balance for unmanned aerial vehicle. Int J Agric \& Biol Eng, 2018; 11(2): 18-26.

\section{Introduction}

China is a large agricultural country and agriculture is vital to people's livelihood as a strong guarantee of the nation's prosperity ${ }^{[1]}$. China's crop diseases and pests have the characteristics of various species, deep degree, high frequency, wide area and serious damage, and are a great threat to crops

Received date: 2017-01-09 Accepted date: 2017-12-13

Biographies: Changling Wang, $\mathrm{PhD}$ candidate, research interests: aerial pesticide application, Email: wc11991@cau.edu.cn; Xiaonan Wang, PhD candidate, research interests: pesticide application, Email: 404694775@qq.com; Zhichong Wang, $\mathrm{PhD}$ candidate, research interests: pesticide droplet atomization, Email: 549422839@qq.com; Shilin Wang, PhD candidate, research interests: pesticide formulation for aerial spraying, Email: 398113027@qq.com; Longlong $\mathbf{L i}, \mathrm{PhD}$ candidate, research interests: orchard precision spraying technology, Email: lizefeng1219@126.com; Jane Bonds, PhD, Senior Consultant, especially food crops. They are also an important influencing factor on the safety of food production, which restrict the promotion of agricultural product quality and the increase of production ${ }^{[2]}$. However, in the production process of China's food crops and other crops at present, plant protection works are still mainly manual and semi-mechanized operations with a large amount of labor and high labor intensity, and the pesticide poisoning incidents during application happen occasionally ${ }^{[3,4]}$.

research interests: agricultural aviation application, Email: jasbonds@gmail.com; Andreas Herbst, $\mathrm{PhD}$, Researcher, research interests: pesticide application technology, Email: andreas.herbst@jki.bund.de; Zhiguo Wang, Chairman, research interests: aerial pesticide application, Email: aywzg339@163.com. *Corresponding author: Xiongkui He, $\mathrm{PhD}$, Professor, research interests: pesticide application. Centre for Chemicals Application Technology, China Agricultural University, No.2 Yuanmingyuan West Road, Haidian District, Beijing 100193, China. Tel: +86-10-62731446, Email: xiongkui@cau.edu.cn. 
Unmanned aerial vehicle (UAV) is of low risk because no pilot is needed. It has high flexibility and doesn't need runway and airport. It is suitable for the works in the paddy field, hills and mountain lands where ground machinery can't be applied. It also has high working efficiency, good spraying quality, strong ability in coping with unexpected disasters, thus reduces the hazard to human and the environmental pollution effectively during the process of pesticide application ${ }^{[5-12]}$

In recent years, China's UAV industry has developed rapidly in the aspects of low-altitude agricultural information remote sensing, agricultural aerial plant protection, and artificial supplementary pollination, etc ${ }^{[13]}$. According to the statistics of Chinese Ministry of Agriculture, there were 178 types of agricultural UAVs in use all over China up to May of 2016 and $95 \%$ of Chinese agricultural aviation technology was applied in aerial plant protection works ${ }^{[14]}$.

With the development of unmanned aerial plant protection, low-altitude and low-volume aerial pesticide application technology is emerging as the research focus. Researchers conducted a series of field tests for aerial pesticide application deposition and drift via various methods, focusing on the effect of the flight parameters on the droplet deposition and the control efficiency of pests and diseases. Fritz et al. ${ }^{[15]}$ assessed aerial spray technologies using an air tractor AT-402B and results indicated that lower spray rates with larger droplet sizes tended to result in greater tracer deposits. Zhang et al. ${ }^{[16]}$ researched the influence of spraying parameters of model 'WPH642' UAV on droplet deposition, using thermal infrared imager to measure the temperatures in the rice canopy before and after spraying and reflect the deposition effect through the temperature change rate. Bae et al. ${ }^{[17]}$ evaluated the effectiveness of a roll balanced helicopter during hovering and cruising spray maneuvers by analyzing flight attitudes and spray patterns. Qiu et al. ${ }^{[18]}$ explored the relationship between spray deposition characteristics and flight height and velocity of an unmanned helicopter of model 'CD-10' and the interaction between two factors. Qin et al. ${ }^{[19]}$ applied model 'HY-B-15L' unmanned helicopter in spraying chlopyrifos in paddy rice fields to control the rice planthopper, and explored the uniformity of the deposition distribution and control efficiency. Zhang et al. ${ }^{[20]}$ researched the droplet deposition effect on citrus trees with different shapes of model ' $3 \mathrm{~W}-L W S-Q 60 S$ ' 4 rotors UAV. Wang et al. ${ }^{[21]}$ studied the distribution characteristics of pesticide spraying droplet deposition of UAV and the effect of flight parameters (flight velocity and altitudes) and parameter accuracy on the deposition distribution.

However, in most of current low-volume and low-altitude aerial pesticide application studies, droplet sampling points were arranged on the ground or at the different heights in the crop's canopy and there was no research reported on the deposition spatial quality balance distribution of UAV pesticide spraying. The choice of droplet sampling material and the accurate measurement of working parameters were always problems in UAV pesticide application test. The water sensitive paper (WSP) was too sensitive to be used in the paddy rice field and other high-humidity environments; petri dish cost much money and a lot time in sampling, which was unsuitable for being used as a droplet collector for field experiment. In the aspect of measuring of flight velocity and altitude, the original method is: record the flying time and distance and take the average velocity calculated as the flight velocity, then estimate the flight altitude by visual inspection via a scale-labeled pole, which is very inaccurate. Besides, the flight speed and altitude could be acquired by airborne global position system (GPS), but the GPS could not meet the accuracy demand of aerial pesticide application research, so the high-accuracy satellite positioning system was in need.

Therefore, a testing method of spatial pesticide spraying deposition quality balance for UAV was proposed in this paper. The detail testing method was: obtain the flight velocity and altitude via the combination of high-accuracy BeiDou navigation satellite system (BNSS) and UAV platform and compare the data of flight parameters from airborne GPS in order to realize precise control; a set of sampling frame of spatial spraying deposition quality balance (SFSSDQB) was invented for collecting the spraying droplets; a set of multi-channel micro-meteorology measurement system (MMMS) was applied for measuring the downwash airflow field of UAV; and then analyze the distribution of spatial spraying deposition quality balance (SSDQB), the downwash airflow field characteristics for UAV pesticide application with accurate flight height and velocity and the relationship between these 2 aspects. Also a series of experiments of model ' 3 WQF80-10' UAV was conducted in order to verify if this testing method was feasible and to study effects of flight directions (ahead and aback), the flight height and the crosswind on the distribution of SSDQB and downwash airflow field. This study can provide a theoretical basis for the designing finalization of UAV, the determination of spraying working parameters and the choice of working conditions and new methods for establishing the standard of spraying deposition and drift test for UAV in crop field, contributing to reducing the droplet drift of aerial pesticide application, and promoting the utilization rate of pesticide.

\section{Testing method of spatial pesticide spraying deposition quality balance for $\mathrm{UAV}$}

\subsection{Materials and instruments}

The experimental system of this method mainly consists of self-invented SFSSDQB, BNSS, MMMS, field weather station and et al.

\subsubsection{SFSSDQB}

In order to achieve the distribution of SSDQB, it's necessary to collect all the droplets in the 4 directions during the pesticide application work. Therefore, a $5 \mathrm{~m} \times 5 \mathrm{~m} \times 2 \mathrm{~m}$ cuboid SFSSDQB (Figure 1a) was built with $85 \mathrm{~m}$ of aluminium bars (Beijing Peace Al Group Co. Ltd.) and $82 \mathrm{~m}$ of $\Phi 2 \mathrm{~cm}$ stainless steel tubes. There were 36 wireropes of $\Phi 2 \mathrm{~mm}$ parallel to the short edge of the SFSSDQB on the 4 sides of the cuboid with the spacing of $50 \mathrm{~cm}$, fixing the deposition sampling wire - $\Phi 2 \mathrm{~mm}$ polyethylene tube (Shenzhen Dongzhenghe Plastics Co. Ltd.). The polyethylene tubes were cut into $0.5 \mathrm{~m}$ per section and put on the SFSSDQB (Figure 1b) with 3 duplicates before the test.

\subsubsection{BNSS}

For high-accuracy global satellite position data, the differential BNSS (ComNav Technology Ltd., Shanghai) was applied in this testing method to record flight height, velocity and track. The system consists of a base station and a mobile station. The base station includes a model 'M300' GNSS (global navigation satellite system) receiver, a model 'AT300' antenna, and a high-power transceiver. M300 GNSS receiver and AT300 antenna are used for receiving satellite signals and differential data and the high-power transceiver transmits the differential signals to the mobile station. Additionally, the mobile station was installed on the UAV to be tested (Figure 2a), including model 'M600' receiver, 
AT300 antenna and $433 \mathrm{MHz}$ data transmission module, and received the satellite signals and differential data and transmitted the positioning data of UAV to $433 \mathrm{MHz}$ data receiver. Data receiver was connected to the laptop via data cable from serial port to universal serial bus (USB) and the flight height and velocity can be displayed and saved in the positioning software system in real time. The post processing static accuracies of the base station were $\pm\left(2.5+1 \times 10^{-6} \mathrm{D}\right) \mathrm{mm}$ (vertical) and $\pm\left(5+1 \times 10^{-6} \mathrm{D}\right) \mathrm{mm}$ (horizontal), where ' $\mathrm{D}$ ' referred to the diameter of the circle with center of the base station; the real time kinematics (RTK) accuracies of the mobile station were $\pm\left(10+1 \times 10^{-6}\right) \mathrm{mm}$ (horizontal) and $\pm\left(20+1 \times 10^{-6}\right)$ (vertical) $\mathrm{mm}$.

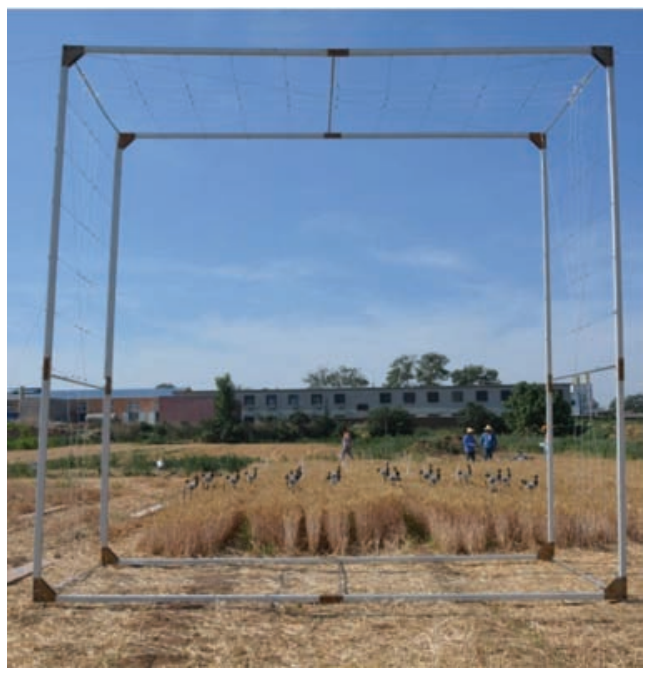

a. Physical photo

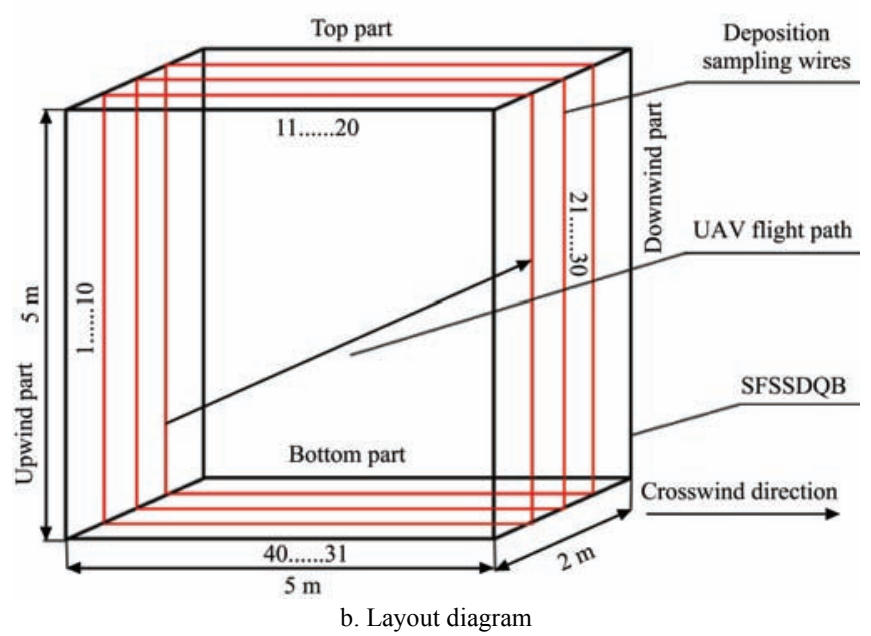

Figure 1 SFSSDQB

\subsubsection{MMMS}

The MMMS was produced by Guangzhou Fumin Measurement \& Control Co., LTD, and consisted of wireless micro-meteorology sensors (WMS, Figure 2b) and intelligent control system (ICS). The WMS, placed in the crop canopy below the UAV flight path, may install 3 blades in 3 spatial directions and a probe and can measure the wind speeds in 3 directions: perpendicular to the flight course $(X)$, perpendicular to the ground $(Y)$ and parallel to the flight course $(Z)$; the WMS can also measure real-time temperature and relative humidity. The ICS contained the wireless micro-meteorology software system, laptop and micro-meteorology data transmission module, emitting control instructions to the WMS and receiving return data of airflow speed. In every test, the sampling time and frequency were $5 \mathrm{~s}$ and $20 \mathrm{~Hz}$ respectively.

\subsubsection{Other materials and instruments}

The meteorological information of the test site was obtained by Zeno-3200 agricultural automatic weather station (Costal Co. Ltd., Figure 2c) and 350-XL analyzer (TESTO Industry Corporation, Germany). Soluble fluorescence tracer Brillantsulfoflavin, (Chroma-Gesellschaft Schmid Co. Ltd., Germany) was prepared into $0.1 \%$ of water solution as spraying liquid before the test and the fluorescence value of eluent was measured by SFM25 spectrofluorometer (KONTRON Instruments) after the test.

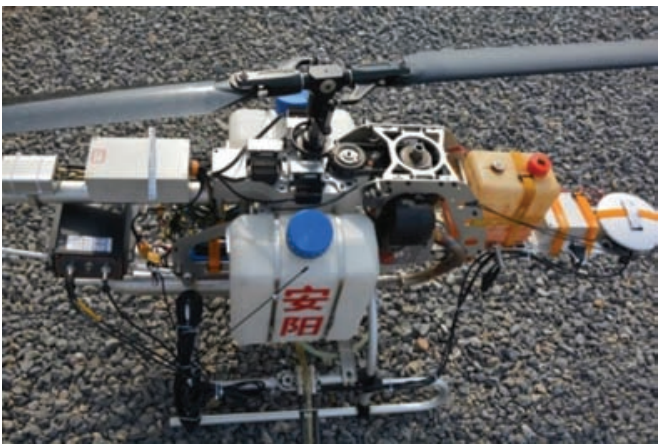

a. Mobile station of BNSS fixed on UAV

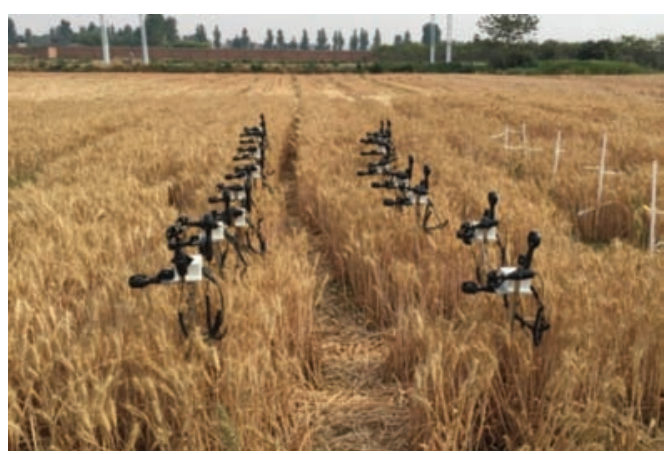

b. Wireless micro-meteorology sensors

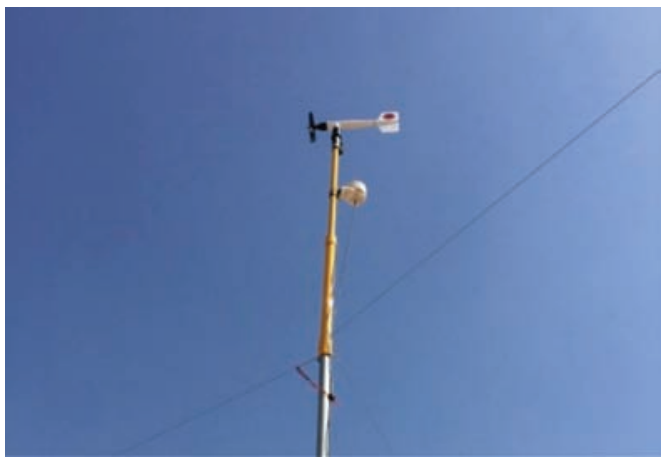

c. Zeno-3200 weather station

Figure 2 Instruments applied in test

\subsection{Testing method}

The SFSSDQB could be placed on the ground as shown in Figure 1 and the flight height and horizontal position when UAV went through the SFSSDQB were set according to UAV's fuselage size and stability. Usually, the safe distance from the edge of frame is more than $1 \mathrm{~m}$. If the SFSSDQB is placed in crop canopy, some holders need to be added beneath the frame so as to make the bottom part of frame locate in the top of crop canopy. The wireless micro-meteorology sensors were arranged in a linear array of 3 directions below the flight path within $5 \mathrm{~m}$ from the frame.

Before the test, the base station of BNSS was fixed within the range of $200 \mathrm{~m}$ from test site and kept still during the whole period of test. Check the UAV to be tested and make sure that the diesel fuel is sufficient and the battery of motor UAV is enough. Meanwhile, fill the prepared $0.1 \%$ of brillantsulfoflavin water 
solution into the tank and fixthe deposition sampling wire on the SFSSDQB. In addition, connect the wireless data receiver for positioning and micro-meteorology data to the laptop and place it within $20 \mathrm{~m}$ 's distance from test site. Turn on the power of WMS and open the wireless micro-meteorology software system. The sensor of TESTO 350-XL analyzer was fixed at the height of $2 \mathrm{~m}$ and the ZENO-3200 weather station was fixed at $6 \mathrm{~m}$, revealing the wind speed and direction in the test site. When the arrangements of experimental field were finished, record precision coordinates of the SFSSDQB and WMSs via the mobile station of BNSS, and then install the mobile station on the UAV for testing. The experimental layout would be changed all the time on the basis of the natural wind direction to confirm the natural wind direction was perpendicular to the UAV flight route.

In every test, the UAV operator controlled the aircraft to take off at $20 \mathrm{~m}$ 's distance from the edge of the field. When it reached the required height and velocity, open the spray system and let the UAV fly into experimental field. Make the UAV go through the frame in the middle, and keep it flying for $5 \mathrm{~m}$ then stop spraying. Avoid passing the experimental field and go back to the starting point. Collect airflow speed data for 5 seconds when the UAV gets to $5 \mathrm{~m}$ 's distance from the WMS. After UAV landed, collect all the deposition sampling wires into valve plastic bags and label them as 'Test No. + Deposition sampling wire No. + Duplicate No.' The sample testing room was established near the test site. When a treatment was finished, the samples of this test would be transported to the testing room and eluted by a certain volume of deionized water. The fluorescence value was measured by SFM25 spectrofluorometer and deposition and deposition rate were figured out. Besides, a blank control group was set up for avoiding the disturbance of experimental materials and other factors.

\subsection{Data analysis}

2.3.1 Deposition and deposition rate

According to the ISO (International Organization for Standardization) standard 24253-1 $1^{[22]}$, the deposition and deposition rate could be calculated by the following equation:

$$
\begin{array}{r}
\beta_{\text {dep }}=\frac{\left(\rho_{\text {smpl }}-\rho_{\text {blk }}\right) \times F_{\text {cal }} \times V_{\text {dii }}}{\rho_{\text {spray }} \times A_{\text {col }}} \\
\beta_{\text {dep } \%}=\frac{\beta_{\text {dep }}}{\left(\beta_{\mathrm{V}} / 100\right)} \times 100
\end{array}
$$

where, $\beta_{\text {dep }}$ is the spraying deposition, expressed in microliters per square centimeter, $\mu \mathrm{L} / \mathrm{cm}^{2} ; \beta_{\mathrm{dep} \%}$ is the deposition rate, $\% ; \beta_{\mathrm{v}}$ is the spray volume, expressed in liters per hectare, $\mathrm{L} / \mathrm{hm}^{2} ; \rho_{\text {smpl }}$ is the fluorimeter reading of the sample; $\rho_{\mathrm{blk}}$ is the fluorimeter reading of the blanks (collector + dilution water); $F_{\text {cal }}$ is the calibration factor; $V_{\text {dii }}$ is the volume of dilution liquid used to dilute tracer from collector, expressed in liters, $\mathrm{L} ; \rho_{\text {spray }}$ is the spray concentration, or amount of tracer solute in the spray liquid sampled at the nozzle, expressed in grams per liter, $\mathrm{g} / \mathrm{L} ; A_{\mathrm{col}}$ is the projected area of the collector for catching the spray deposition, expressed in square centimeters, $\mathrm{cm}^{2}$.

\subsubsection{Coefficient of variation (CV) of deposition rate}

The $\mathrm{CV}$ was used as a measure of the droplet distribution uniformity ${ }^{[23]}$, according to the equation below:

$$
\mathrm{CV}=\frac{S}{\bar{X}} \times 100, S=\sqrt{\frac{\sum_{i=1}^{n}\left(X_{i}-\bar{X}\right)^{2}}{n-1}}
$$

where, $S$ is the standard deviation, $\%) ; X_{i}$ is the deposition rate per unit zone in the sampling wire, $\% ; \bar{X}$ is the average deposition rate per unit zone in the sampling wire, $\%$; and $n$ is the total number of sampling wires.

2.3.3 Weighted mean deposition rate

In order to research the effect of crosswind speed on the distribution of SSDQB, it's necessary to find an evaluation index for indicating the centralized tendency of droplet distribution. When analyzing the deposition at the bottom part of SFSSDQB, a type of weighted average was adopted and increasing weights were assigned to all deposition sampling wires to judge the centralized tendency of deposition distribution according to the distance away from the downwind part - the weight rose with decrease of the distance. Therefore, 10 different weights ' $1-10$ ' were assigned to the deposition sampling wires in the order from No. 40 to No. 31 , and the calculated weighted average was called weighted mean deposition rate. The higher the mean deposition rate is, the more droplets will deposit near the downwind part. The weighted mean deposition rate could be calculated by the equation below:

$$
\bar{X}^{\prime}=\frac{\sum_{i=1}^{n} X_{i} f_{i}}{\sum_{i=1}^{n} f_{i}}
$$

where, $\bar{X}$ ' is the weighted mean deposition $\operatorname{rate}(\%) ; f_{i}$ is the weight.

\section{$3 \quad$ Field test of spatial pesticide spraying deposition quality balance for model ' $3 \mathrm{WQF80-10}$ ' UAV}

\subsection{Test site}

The test was conducted in the wheat planting base $\left(114^{\circ} 39.713^{\prime} \mathrm{E}, 36^{\circ} 15.599^{\prime} \mathrm{N}\right)$ of Anyang Quanfeng Aviation Plant Protection Technology Co., Ltd., Henan province from June to July, 2015. Conditions of the wheat field were shown as follows: breed, rumai 0319 ; line spacing, $20 \mathrm{~cm}$; plant height, $60 \mathrm{~cm}$; density, 20 plants $/ \mathrm{m}^{2}$; growth period is in maturation stage.

\subsection{Model of UAV}

The tested UAV was model ' $3 \mathrm{WQF} 80-10$ ' diesel single-rotor plant protection unmanned helicopter with $10 \mathrm{~L}$ of tank and 2-3 m of spray swath at height of 2-3 m, controlled by the operator. Two flat standard nozzles of LU 120-02 are installed on the helicopter and the flow rate of a single nozzle are $0.56-1.03 \mathrm{~L} / \mathrm{min}$

\begin{tabular}{|c|c|c|c|c|c|c|c|c|c|}
\hline Type & $\begin{array}{l}\text { Fuselage } \\
\text { length } / \mathrm{mm}\end{array}$ & $\begin{array}{c}\text { Rotor } \\
\text { diameter } / \mathrm{mm}\end{array}$ & $\begin{array}{c}\text { Boom } \\
\text { width } / \mathrm{mm}\end{array}$ & $\begin{array}{c}\text { Fuselage } \\
\text { height } / \mathrm{mm}\end{array}$ & $\begin{array}{c}\text { Total } \\
\text { weight } / \mathrm{kg}\end{array}$ & $\begin{array}{c}\text { Number of } \\
\text { nozzle }\end{array}$ & $\begin{array}{c}\text { Nozzle } \\
\text { spacing/mm }\end{array}$ & $\begin{array}{l}\text { Type of } \\
\text { nozzle }\end{array}$ & $\begin{array}{l}\text { Flow rate } \\
/ \mathrm{L} \cdot \mathrm{min}^{-1}\end{array}$ \\
\hline 3WQF80-10 & 2920 & 1800 & 1100 & 750 & 35 & 2 & 1100 & LU 120-02 & 1.8 \\
\hline
\end{tabular}
within the spraying pressure range of $0.15-0.50 \mathrm{MPa}$. The primary technical parameters were shown in Table 1.

\section{Table 1 Primary technical parameters of model 3WQF80-10 UAV}

\subsection{Experimental treatment}

Field experiments were carried out according to the designed testing method of spatial pesticide spraying deposition quality balance for UAV. The layout of experimental field is shown as
Figure 3a. The SFSSDQB was placed along the edge of wheat field and the WMSs were distributed within the field. The wireless micro-meteorology sensors were fixed at the top of wheat canopy in linear array with spacing of $0.7 \mathrm{~m}$ and line spacing of 
$1.5 \mathrm{~m}$. There were 2 lines and 16 sensors in total, whose serial numbers were '1-16'. The serial numbers of deposition sampling wires started from the upwind part, 1-10; top part, 11-20; downwind part, 21-30; bottom part, 31-40 (Figure 1b), convenient for arrangement and collection of samples and data analysis.

Figure $3 \mathrm{~b}$ shows the field test site of model ' $3 \mathrm{WQF} 80-10$ ' unmanned helicopter. The flight path of UAV was the center line of the horizontal of the frame. 4 parts of tests were set: 1) in the test of distribution of SSDQB (Test 1-3), the flight height was $2.5 \mathrm{~m}$ and the velocity was $5.0 \mathrm{~m} / \mathrm{s}$, and there were 3 duplicates; 2 ) in the test of flight direction (Test 4-7), the flight height was $3.0 \mathrm{~m}$ and the velocity was $5.0 \mathrm{~m} / \mathrm{s}$, and there were respectively 2 forward duplicates and 2 backward duplicates; 3 ) in the test of flight height (Test 8-13), 6 different heights of 2.0-3.5 m were tested at a velocity of $5.0 \mathrm{~m} / \mathrm{s} ; 4)$ in the test of crosswind (Test 14-18), there were 5 treatments of diverse crosswind speeds at the height of $3 \mathrm{~m}$ and the velocity of $5.0 \mathrm{~m} / \mathrm{s}$.

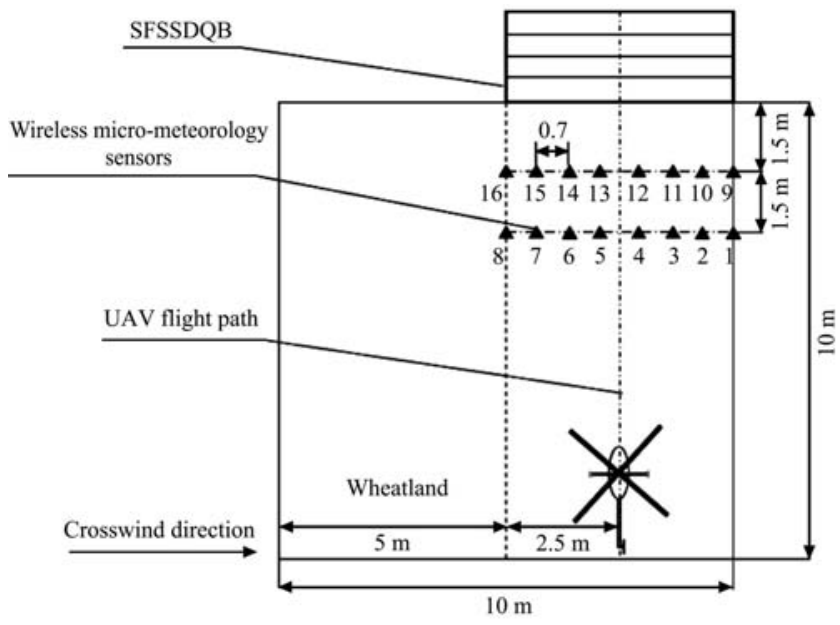

a. Experimental field layout diagram

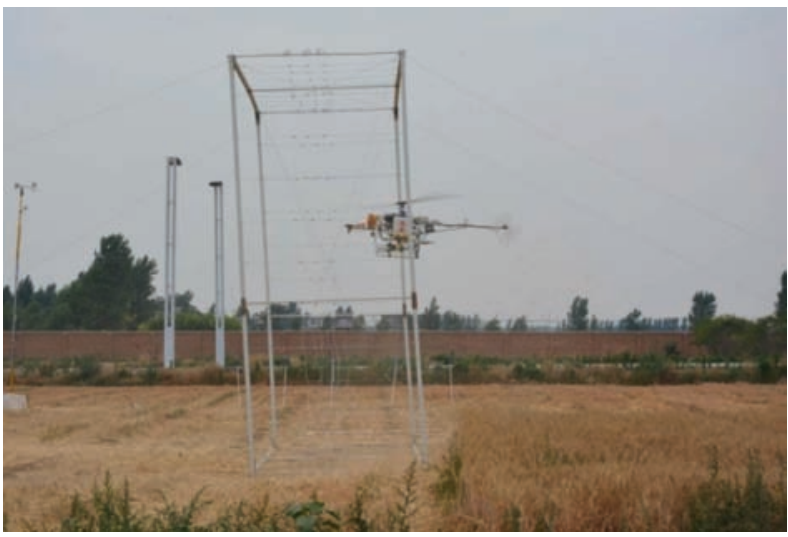

b. Field test site

Figure 3 Field test

\section{$4 \quad$ Results and discussion}

\subsection{Actual flight parameters}

The test number, flight height, flight velocity and natural crosswind speed measured by the BNSS and MMMS are shown in Figure 2.

Table 2 Actual flight height and velocity

\begin{tabular}{|c|c|c|}
\hline Test No. & Flight height $/ \mathrm{m}$ & Flight velocity $/ \mathrm{m} \cdot \mathrm{s}^{-1}$ \\
\hline 1 & 2.49 & 5.3 \\
\hline 2 & 2.95 & 4.7 \\
\hline 3 & 3.08 & 5.2 \\
\hline 4 & 3.05 & 5.1 \\
\hline 5 & 3.08 & 4.8 \\
\hline 6 & 3.00 & 4.8 \\
\hline 7 & 2.95 & 5.0 \\
\hline 8 & 2.00 & 4.9 \\
\hline 9 & 2.57 & 4.8 \\
\hline 10 & 2.70 & 5.0 \\
\hline 11 & 3.08 & 5.3 \\
\hline 12 & 3.11 & 4.7 \\
\hline 13 & 3.23 & 4.8 \\
\hline 14 & 2.95 & 5.0 \\
\hline 15 & 3.09 & 4.7 \\
\hline 16 & 3.08 & 5.1 \\
\hline 17 & 2.97 & 4.8 \\
\hline 18 & 3.00 & 4.9 \\
\hline
\end{tabular}

\subsection{Distribution of SSDQB and downwash flow field}

As shown in Table 3, the ratio refers to ratio between the summary of 10 deposition rates of one part of SFSSDQB and the summary of all deposition rates of the whole frame. The deposition distribution of test 1 and 2 was similar but the difference in between test 1, 2 and test 3 was obvious because of the varied crosswind, it's needed to explore the effect of the crosswind speed on deposition distribution. We can calculate that: When the average wind speed was $0.9 \mathrm{~m} / \mathrm{s}$, the average temperature was $31.5^{\circ} \mathrm{C}$ and the average relative humidity was $34.1 \%$, the average distribution ratios of spraying deposition for model ' $3 \mathrm{WQF} 80-10$ ' UAV on the upwind part, the top part, the downwind part and the bottom part were $4.4 \%, 2.3 \%, 50.4 \%$ and $43.7 \%$ respectively. The droplets mainly deposited on the downwind and bottom part and there were few droplets depositing on the upwind and the top part.

The speed of downwash airflow of UAV's rotor in flight was measured in directions of $X$ (perpendicular to the flight course), $Y$ (perpendicular to the ground) and $Z$ (parallel to the flight course) via the MMMS, and Figure 4 shows the distribution of peak value of rotor's downwash airflow speed in 3 directions and all the results are jointly determined by the natural wind and rotor's downwash airflow. According to the diagram of wireless micro-meteorology sensors, the natural crosswind direction was from No. 8 to No. 1 and UAV flight path was above the central line between sensor No. 4 and No. 5 .

Table 3 Maximum, mean and ratio of different parts in SFSSDQB

\begin{tabular}{|c|c|c|c|c|c|c|c|c|c|c|c|c|c|}
\hline \multirow{2}{*}{ Test No. } & \multirow{2}{*}{$\begin{array}{l}\text { Crosswind } \\
\text { speed } / \mathrm{m}^{-1} \mathrm{~s}^{-1}\end{array}$} & \multicolumn{3}{|c|}{ Upwind } & \multicolumn{3}{|c|}{ Top } & \multicolumn{3}{|c|}{ Downwind } & \multicolumn{3}{|c|}{ Bottom } \\
\hline & & $\operatorname{Max} / \%$ & Mean/\% & Ratio/\% & $\operatorname{Max} / \%$ & Mean $/ \%$ & Ratio/\% & $\operatorname{Max} / \%$ & Mean/\% & Ratio/\% & $\operatorname{Max} / \%$ & Mean/\% & Ratio/\% \\
\hline 1 & 1.20 & 1.7 & 1.3 & 1.8 & 1.8 & 1.5 & 2.1 & 91.3 & 40.8 & 57.3 & 41.5 & 27.6 & 38.8 \\
\hline 2 & 1.13 & 3.5 & 2.6 & 3.6 & 2.0 & 1.0 & 3.6 & 83.9 & 47.2 & 64.8 & 30.6 & 22.0 & 30.3 \\
\hline 3 & 0.40 & 13.1 & 5.8 & 7.9 & 2.0 & 0.9 & 1.2 & 38.5 & 21.4 & 29.1 & 77.0 & 45.7 & 61.9 \\
\hline
\end{tabular}

Note: The environmental parameters during the test: average temperature was $31.5^{\circ} \mathrm{C}$, average relative humidity was $34.1 \%$. 


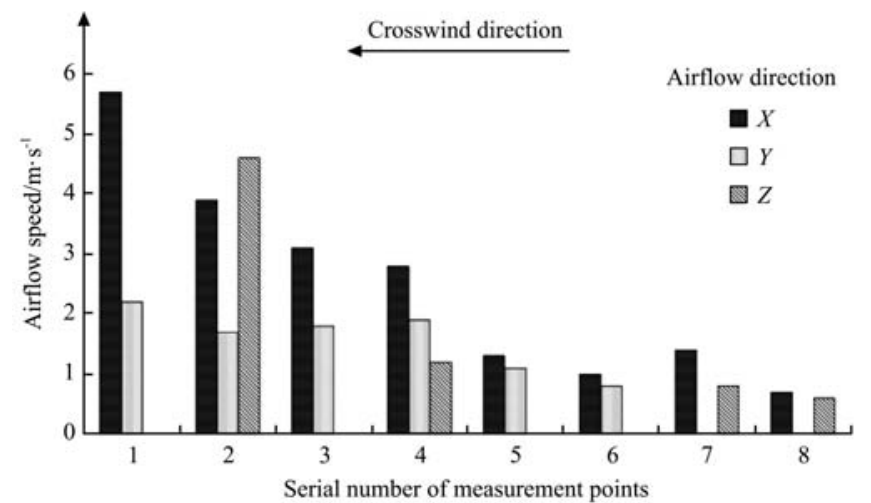

Figure 4 Airflow speed distribution of downwash flow of model '3WQF80-10' UAV

With regard to the ' $3 \mathrm{WQF} 80-10$ ' single-rotor UAV, the wind speed in direction $X$ was faster than that in direction $Y$, and the wind speed in direction $Y$ was faster than that in direction $Z$ (Figure 4). The downwash airflow in the direction perpendicular to the ground was the strongest, having greater influence on spraying deposition than the direction $X$ and $Z$. It can be seen from Figure 4 , peak values of airflow speed in direction $X$ became stronger from the upwind part to the downwind part and the airflow field at the spot of sensor No.1 was greatest. That's because when UAV flied along the central line between sensor 4 and 5, the wind generated by UAV would flow to 2 opposite directions from the flight path: one direction was downwind and there would be a stronger wind field due to the superposition of natural crosswind and it; the other was the upwind direction and the counteraction of reversed natural wind would contribute to the low wind speed and weak wind field in that section. Moreover, considering the results of the deposition rate on the SFSSDQB, there were more droplets depositing on the downwind part where the airflow field was stronger, which meant the distribution of rotor's downwash airflow field approximately conformed to the deposition distribution on the sampling frame.

4.3 Effect of flight direction on spraying deposition distribution

In the process of UAV's practical spraying work in crop field, the route is mostly an ' $m$ ', meaning that the UAV flies forwards to the far end from the controller's side and decelerates to hovering, then the UAV moves parallel to the next line and keeps the nose ahead flying from the far end back to the near end, and then moves parallel again to the next round of work. In the process of flying forwards and backwards, the nose of the unmanned helicopter faces forwards and backwards respectively. For the single-rotor UAV, its construction differs from the symmetrical type of multi-rotor $\mathrm{UAV}$, so it is necessary to analyze if the flight direction (ahead and aback) would have an influence on the distribution of SSDQB. As shown in Figure 3, there were 4 tests conducted at the height of $3.0 \pm 0.1 \mathrm{~m}$ and the velocity of $5.0 \pm 0.2 \mathrm{~m} / \mathrm{s}$, where test 4 and 5 were forward while test 6 and 7 were backward. Also, the deposition rate ratio of these 4 tests is displayed in Table 4, and Figure 5 shows the distribution of SSDQB of 2 different flight directions. The results of Table 4 and Figure 5 indicate that: when the UAV went forwards, there were few droplets depositing on the sampling wire 1-20; the maximum deposition rate was within the range from No. 25 to 30 (the maximum of test 4 was $128.3 \%$ of No. 28 and maximum of test 5 was $135.2 \%$ of No. 25 ) and the deposition ratio on the downwind part was the most, up to $60 \%$; the deposition rate on the bottom was basically less than $60 \%$ and the distribution ratio was in the scope from $30 \%$ to $40 \%$ as well. While the UAV flies backwards, a few deposited droplets on the sampling wire 1 10 of the upwind part could be measured and the ratio was in the range from 10 to $20 \%$; the max of deposition rate was on the bottom (the maximum of test 6 was $124.1 \%$ of No. 34 and max of test 7 was $93.2 \%$ of No. 36), and the ratio of this part was also the most, more than $50 \%$. Therefore, the difference caused by these 2 flight directions mainly focuses on the following 2 aspects: how many droplets deposit on the upwind part and where most of droplets deposit - downwind or bottom. During the process of UAV spraying work in field, the optimal application situation is that pesticide droplets sprayed from nozzles move down vertically and deposit directly on the target plant. These downward droplets have the lowest drift rate but the highest utilization; droplets move sidewise have different movement loci - those large-size droplets may still attach to leaves of the crops, yet under the coaction of the natural wind and the downwash airflow other small-size droplets is much easier to drift to non-target plants and the environment or even evaporate directly in the air, contributing high drifting ratio, low utilization phytotoxicity and pollution. Although flying backwards could lead spraying droplets to deposit on the upwind part of the SFSSDQB, its uppermost deposition part was still the bottom. As a result, considering the distribution ratio of backward flight was as much as $60 \%$, the backward direction was better and able to reduce the drift and promote the deposition rate on the target plant and the utilization of pesticide.

Table 4 Deposition rate ratio of different parts in tests of effect of flight direction on droplet deposition distribution

\begin{tabular}{cccccc}
\hline Test No. & Flight direction & Upwind/\% & Top/\% & Downwind/\% & Bottom/\% \\
\hline 4 & Forwards & 1.8 & 2.1 & 57.3 & 38.8 \\
5 & Forwards & 3.6 & 1.3 & 64.8 & 30.3 \\
6 & Backwards & 12.7 & 2.6 & 24.4 & 60.4 \\
7 & Backwards & 19.4 & 0.8 & 28.7 & 51.1 \\
\hline
\end{tabular}

Note: The environmental parameters during the test: Average crosswind speed is $1.2 \mathrm{~m} / \mathrm{s}$, average temperature is $30.5^{\circ} \mathrm{C}$, average relative humidity is $36.9 \%$.

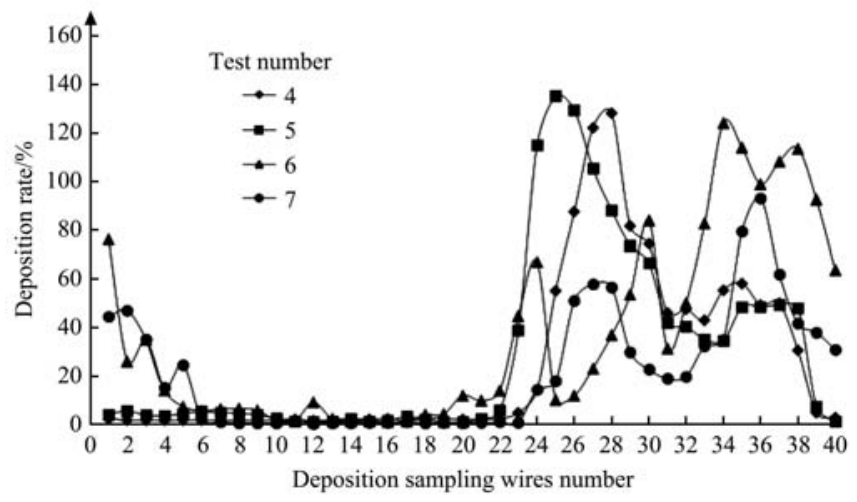

Figure 5 Spatial spray deposition distribution of UAVs when flying forwards and backwards

The distribution of downwash airflow speed in the direction $Y$ is shown in Figure 6. It was seen that when the helicopter went backwards the peak value of airflow speed in $Y$ was generally higher than forwards, meaning its influence of rotor's downwash airflow in $Y$ was more than the forward flight. While the airflow perpendicular to the ground has the most obvious effects on droplets' downward movement towards crops, the droplets deposited more on the bottom part as the single-rotor UAV flies backwards through the sampling frame. Besides, the airflow speeds of the measurement sensor No. 7 and 8 were relatively slow, turning out that the downwash airflow had a weaker effect on 
pushing down the spraying droplets. Furthermore, it was easier to result in the sideward movement of droplets, which could also explain why a few droplets deposited on the upwind. It is analyzed that as this model of unmanned helicopter flies forwards, the direction of the rotor's downwash airflow is not vertically downward but between the vertical and the direction of the tail under the influence of the opposite outside airflow. And the airflow passes the fuselage and will be blocked by the fuselage and change the direction, so the downwash airflow pushing down droplets would be weakened greatly. Nonetheless, when the UAV flies backwards, the direction of the downwash airflow was downward between the vertical and the direction of the fuselage, and the probability that the airflow will be blocked by the fuselage reduces obviously, generating a stream of strong downward airflow passed the boom on the bottom of the fuselage, and helping the spraying droplets move down and reach the surface of the plant. Overall, at the height of $3.0 \pm 0.1 \mathrm{~m}$, the velocity of $5.0 \pm 0.2 \mathrm{~m} / \mathrm{s}$ and the crosswind speed of $1.2 \mathrm{~m} / \mathrm{s}$, the flight directions of ahead and back had an impact on droplet deposition distribution. The working effect of flying backwards was better than flying forwards; In addition, the difference of the distribution of 2 flight directions was due to the different effects of the downwash airflow field of forward and backward flights on droplets, which was caused by the asymmetric structure of the single - rotor UAV.

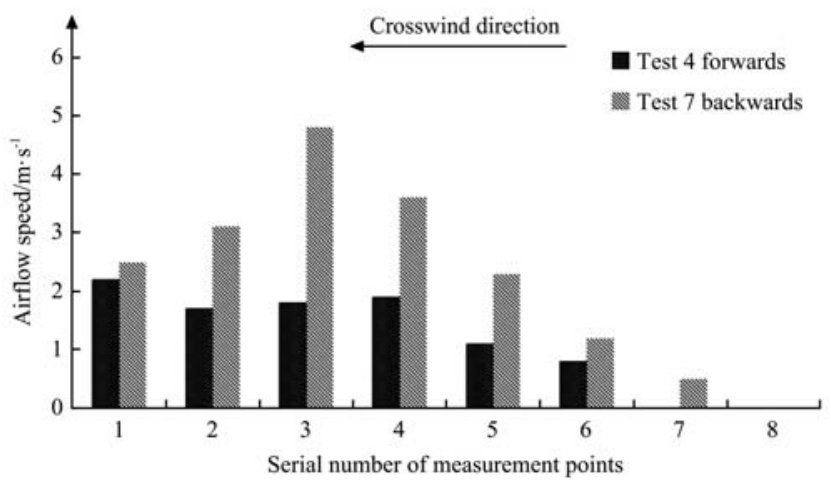

Figure 6 Airflow speed distribution of downwash flow in the direction perpendicular to the ground when flying forwards and backwards

\subsection{Effect of flight altitude on spraying deposition distribution}

The flight height is an important parameter for UAV spraying work. Previous researches ${ }^{[15-21]}$ show that there were effects in various flight heights on the spraying deposition, penetrability in canopy and distribution uniformity. Hence, in order to explore the regularities of distribution of different flight heights, 6 tests were conducted and the $\mathrm{CV}$ on the bottom part of SFSSDQB and deposition distribution ratio were shown in Figure 4.

Table $5 \mathrm{CV}$ of deposition rate on bottom part and deposition rate ratio of different parts in tests of effect of flight height on droplet deposition distribution

\begin{tabular}{ccccccc}
\hline $\begin{array}{c}\text { Test } \\
\text { No. }\end{array}$ & $\begin{array}{c}\text { Flight } \\
\text { height/m }\end{array}$ & CV/\% & $\begin{array}{c}\text { Upwind } \\
/ \%\end{array}$ & $\begin{array}{c}\text { Top } \\
/ \%\end{array}$ & $\begin{array}{c}\text { Downwind } \\
/ \%\end{array}$ & $\begin{array}{c}\text { Bottom } \\
/ \%\end{array}$ \\
\hline 8 & 2.00 & 80.2 & 1.1 & 1.4 & 18.9 & 78.6 \\
9 & 2.57 & 55.2 & 5.6 & 4.3 & 19.9 & 70.3 \\
10 & 2.70 & 52.7 & 9.7 & 0.9 & 39.0 & 50.4 \\
11 & 3.08 & 48.7 & 2.8 & 1.1 & 56.6 & 39.5 \\
12 & 3.11 & 46.5 & 5.6 & 1.3 & 63.9 & 29.2 \\
13 & 3.23 & 44.4 & 7.9 & 2.2 & 51.9 & 38.1 \\
\hline
\end{tabular}

meters during the test: $0.80 \mathrm{~m} / \mathrm{s}$; average temperature was $28.4^{\circ} \mathrm{C}$; average relative humidity was $41.6 \%$.
The CV of deposition rate of the tracer Brillantsulfoflavin on 4 parts of the sampling frame was calculated according to the Equation (3), and then the linear regression analysis was applied between the $\mathrm{CV}$ of 4 parts in tests and the flight height. It turned out that the linear regression equation of the bottom part was $y=-27.327 x+130.64$ with the coefficient of determination of 0.9178 There was no significant linear relationship between the CV and the height for the other parts of the SFSSDQB. Therefore, the bottom results were primarily analyzed and the scatter diagram was plotted (Figure 7). At the height from 2.0 to $3.5 \mathrm{~m}$ and the velocity of $5.0 \pm 0.3 \mathrm{~m} / \mathrm{s}$, with the increase of the flight height, there was a linear decrease of the $\mathrm{CV}$ of the deposition rate, indicating increasing the flight height could reduce the dispersion degree of droplet deposition and promote the uniformity of the droplet distribution. Meanwhile, with the increase of the flight height, the deposition distribution ratio of the bottom part lowered from $78.6 \%$ to less than $40 \%$; the ratio of the downwind part appeared an obvious rising trend; and the ratio of the upwind part increased slightly. The ratio of the top showed no significant change.

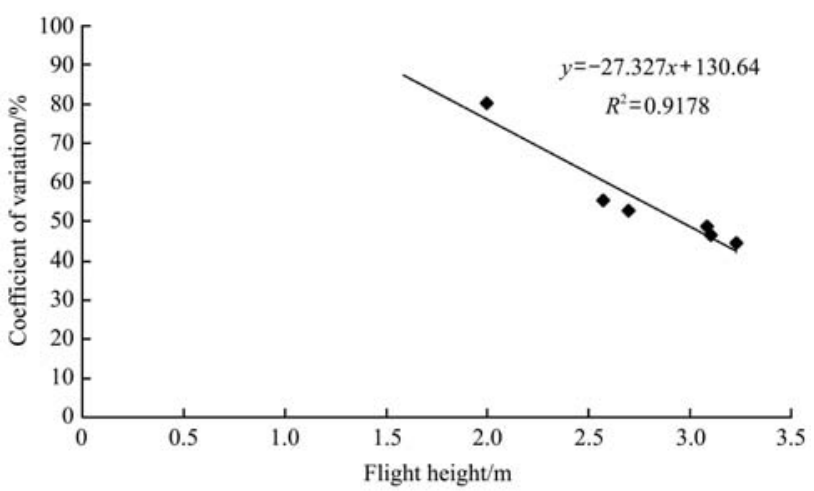

Note: In tests of flight height, the flight velocity was $5.0 \mathrm{~m} / \mathrm{s}$; the flight direction was forwards; the crosswind speed was $0.80 \mathrm{~m} / \mathrm{s}$; average temperature was $28.4^{\circ} \mathrm{C}$; average relative humidity was $41.6 \%$.

Figure 7 Relationship between the CV of deposition rate on the bottom of spatial spray deposition sampling frame and flight height

Considering the distribution in $Y$ of rotor's downwash airflow speed at different heights, it can been seen that at the flight height of $2.00 \mathrm{~m}$, the downwash airflow speed was quite fast and the airflow field was relatively strong. Along with the raise of the flight height, the airflow field in the direction perpendicular to the ground weakened significantly. As the flight height was greater than $3 \mathrm{~m}$, the downwash airflow speed in direction $Y$ almost cannot be measured and this result agreed basically with that of downwash airflow field of single-rotor motor UAV in earlier studies ${ }^{[24]}$. It showed that the change of the flight height didn't only have an impact on the $\mathrm{CV}$ of the deposition distribution of the bottom part but also had a close relationship with the droplet drift characteristics. With increased distance between the downwash airflow and the rotor, the airflow will change the direction from vertically downward movement to horizontal ${ }^{[25]}$. Thus the higher the height is, the weaker the rotor's downwash airflow in the top of canopy is, and more easily spraying droplets will drift with the crosswind. The results above demonstrate that the method for adjusting the $\mathrm{CV}$ of droplets distribution via the flight height was a two-edged sword. Because increasing the height blindly can't improve the spraying effect of this model of UAV all the time but flying too high would weaken the pushing effect on droplets of the downwash airflow and aggravate the droplet drift, thus reduce the utilization of pesticide. Hence, the crop characteristics, the field situation, the weather condition and other factors need to be taken 
into overall consideration during the field spraying work of UAV.

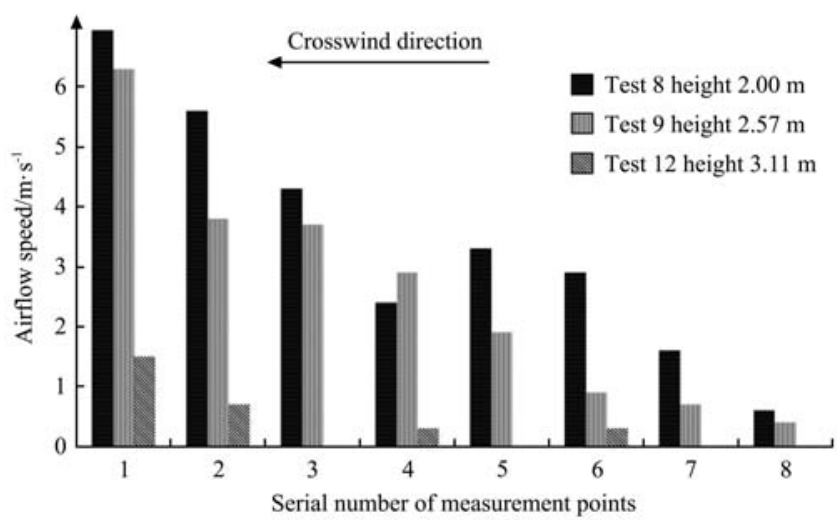

Figure 8 Wind speed distribution of downwash flow in direction perpendicular to the ground of different flight heights

\subsection{Effect of crosswind speed on spraying deposition} distribution

Aerial spraying work for plant protection is easy to be affected by airflow strength, airflow direction and other factors, bringing about overlapping spray, leaky spray and droplet drift $^{[26]}$. Regarding the model ' 3 WQF80-10' UAV with 2 'LU120-02' nozzles, the size of droplets generated was from 120 to $160 \mu \mathrm{m}$ and they were very likely to be influenced by the airflow, so it's required to study the effect of the crosswind speed on the distribution of SSDQB. There were 5 tests at the height of $3.0 \mathrm{~m}$, the velocity of $5.0 \mathrm{~m} / \mathrm{s}$ and various crosswind speeds, weighted mean deposition rate and deposition rate ratio of different parts are shown in Table 6 .

Table 6 Crosswind speed, weighted mean deposition rate and deposition rate ratio of different parts in tests of effect of crosswind speed on droplet deposition distribution

\begin{tabular}{ccccccc}
\hline $\begin{array}{c}\text { Test } \\
\text { No. }\end{array}$ & $\begin{array}{c}\text { Crosswind } \\
\text { speed } / \mathrm{m}^{-1} \mathrm{~s}^{-1}\end{array}$ & $\begin{array}{c}\text { Weighted mean } \\
\text { deposition rate/\% } \%\end{array}$ & $\begin{array}{c}\text { Upwind } \\
/ \%\end{array}$ & $\begin{array}{c}\text { Top } \\
/ \%\end{array}$ & $\begin{array}{c}\text { Downwind } \\
/ \%\end{array}$ & $\begin{array}{c}\text { Bottom } \\
/ \%\end{array}$ \\
\hline 14 & 0.61 & 29.1 & 2.4 & 3.6 & 39.1 & 54.9 \\
15 & 1.03 & 40.0 & 3.6 & 1.3 & 56.8 & 40.6 \\
16 & 1.19 & 45.8 & 2.1 & 3.7 & 57.3 & 36.9 \\
17 & 2.17 & 49.3 & 17.6 & 2.4 & 39.9 & 40.2 \\
18 & 5.50 & 86.6 & 11.0 & 1.5 & 58.9 & 28.6 \\
\hline
\end{tabular}

Note: The environmental parameters during the test: Average temperature was $27.5^{\circ} \mathrm{C}$, average relative humidity was $50.3 \%$.

The weighted mean deposition rate of 4 parts of the sampling frame was calculated via the Equation (4). The linear regression analysis between the weighted mean deposition rate and the crosswind speed was conducted and the results showed that: the linear regression equation of the bottom part was $y=10.785 x+$ 27.499 with the coefficient of determination of 0.9684 ; there was not any significant linear relationship between those 2 variables for the other parts of the SFSSDQB. As shown in Figure 9, with the increase of the crosswind speed, there was a linear increase of the weighted average, indicating the droplets distribution leans to the downwind part gradually because of the increase of the weight according to the crosswind direction from upwind to downwind. In addition, at the height of $3.0 \pm 0.1 \mathrm{~m}$ and the velocity of $5.0 \pm$ $0.3 \mathrm{~m} / \mathrm{s}$, when the crosswind speed increased, the deposition rate ratio of the bottom part decreased and the ratio of side parts especially the downwind part rose slowly, resulting from that the deposition concentrated on the downwind part as well. At the same time, it turned out that from the distribution of downwash airflow speed in direction $Y$ (Figure 10), with increasing crosswind speed, measured airflow speed in every wireless micrometeorology sensor had a decreasing tendency and downwash airflow field weakened gradually. It was because that the direction of downwash airflow was changed and the intensity of the airflow in direction $Y$ was reduced under the influence of the enhanced crosswind. Droplets tended to move around instead of moving downward and this kind of distribution of airflow verified that the crosswind had an adverse impact on the droplet deposition obviously. Results above show that: the effects of the crosswind on the droplet deposition are mainly to reduce the intensity of the downwash airflow field in the direction perpendicular to the ground and to let the droplet deposition concentrate on the downwind side, reducing the ratio of deposited droplets and increasing the number of drifted droplets.

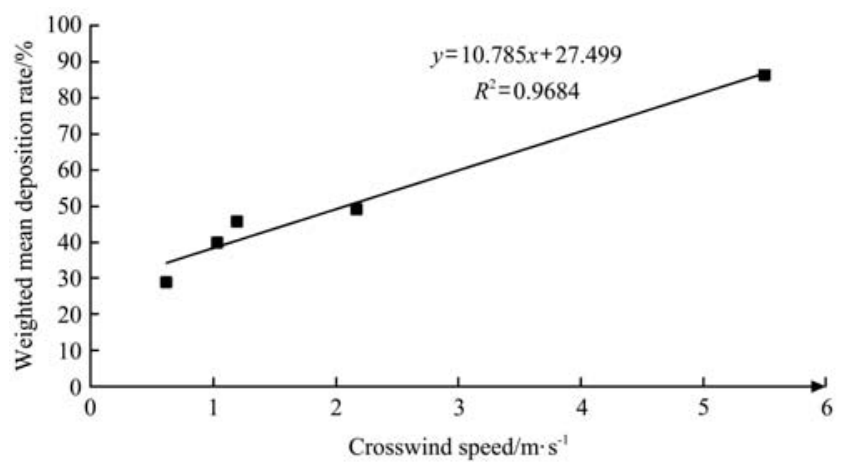

Note: In tests of crosswind speed, the flight velocity was $5.0 \mathrm{~m} / \mathrm{s}$; the fligh height was $3.00 \mathrm{~m}$; the flight direction was forwards; average temperature was $27.5^{\circ} \mathrm{C}$; average relative humidity was $50.3 \%$.

Figure 9 Relationship between weighted mean deposition rate on bottom of SFSSDQB and crosswind speed

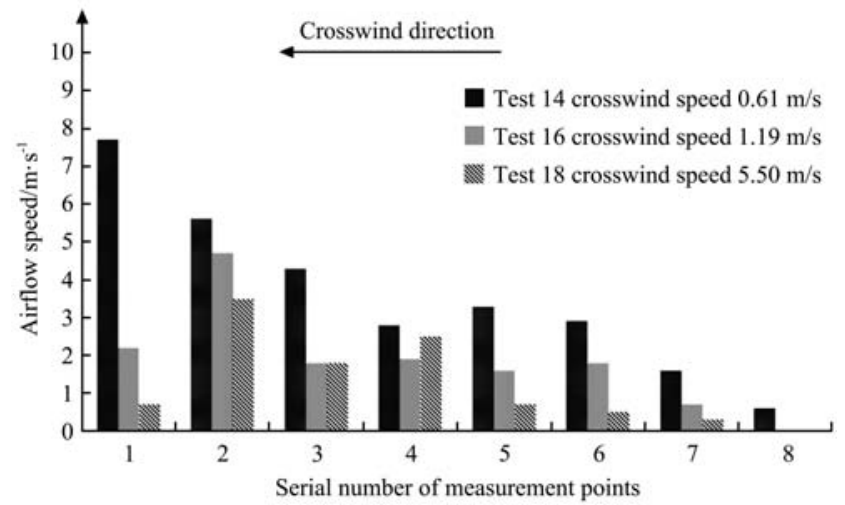

Figure 10 Distribution of downwash airflow speed in direction perpendicular to the ground of different crosswind speeds

\section{Conclusions}

This study proposed a novel testing method of spatial pesticide spraying deposition quality balance for UAV. The method was shown as below: obtain the flight velocity and altitude via the combination of high-accuracy BNSS and UAV platform and compare the data of flight parameters from airborne GPS; a set of SFSSDQB was invented for collecting the spraying droplets; a set of MMMS was applied for measuring the downwash airflow field of the unmanned helicopter's rotor. The testing method was also used to research the distribution of SSDQB in different directions (ahead and aback) and at different flight heights and crosswind speeds before drawing the following conclusions:

1) The invented testing method of spatial pesticide spraying deposition quality balance for UAV is feasible, accurate and reliable, providing a new testing and research method for droplet 
deposition distribution of UAV.

2) Through flight parameters monitored by the BNSS, spatial droplet distributions of pesticide application of UAV in different directions at the accurate flight height and velocity were acquired for the first time, and deposited droplets were mainly located on the downwind and bottom part and there were few droplets on the top and upwind part.

3) For the model ' $3 \mathrm{WQF} 80-10$ ' UAV, 2 opposite flight directions had an obvious impact on the distribution of SSDQB. Flying backwards had better working effect and the deposition rate ratio of the bottom part of backward flight can be up to $60 \%$. And there is a linear negative correlation between the $\mathrm{CV}$ of the bottom part and the flight height and the coefficient of determination was 0.9178 , which means that the deposition distribution becomes more uniform with the increase of height. There was a linear positive correlation between weighted mean deposition rate and crosswind speed and the coefficient of determination was 0.9684 , which shows the deposition distribution gets more concentrated towards the downwind part with the rise of the crosswind speed. Moreover, the weaker the downwash airflow field is, the more severe the degree of the droplets drift is.

Regardless of the flight direction, the flight height and the crosswind, the influences on the spraying droplets of the single-rotor UAV are to alter the intensity of the downwash airflow field in the direction perpendicular to the ground and to weaken the downward pushing effect of the airflow on droplets. Therefore, the downwash airflow field of UAV has a key impact upon the movement of droplets of UAV aerial spraying work. And in the process of the design, manufacture and field application, it's needed to consider the effects of fuselage structure, boom position, nozzle type, flight parameters and meteorological condition sufficiently on the downwash airflow to reduce the negative effects to the maximum level.

\section{Acknowledgements}

This work was supported by Special Fund for Agro-scientific Research in the Public Interest (201503130) and National Science Fund Projects (31470099). Many thanks to Xuesong $\mathrm{Xu}$ and Xijun Deng of Anyang Quanfeng Aviation Plant Protection Technology Co., Ltd. for their contribution to the work.

\section{[References]}

[1] Fan Q N. The Research on the Pesticide Spray System Using for the Mini Unmanned Helicopter. Nanjing: Nanjing Forestry University, 2011. (in Chinese)

[2] Shao Z R, Guo Y W. Situation and strategies of spraying facilities and pesticide application technology in China. Plant Protection, 2006; 32(2): 5-8. (in Chinese)

[3] Zhou Z Y, Zang Y, Luo X W, et al. Technology innovation development strategy on agricultural aviation industry for plant protection in China. Transactions of the CSAE, 2013; 29(24): 1-10. (in Chinese)

[4] He X K. Improving severe draggling actuality of plant protection machinery and its application techniques. Transactions of the CSAE, 2004; 20(1): 13-15. (in Chinese)

[5] Ma X Y, Wang Z G, Jiang W L, et al. Analysis of current status and application prospects of unmanned Aerial Vehicle Plant protection technology in cotton field in China. China Cotton, 2016; 43(6): 7-11. (in
Chinese)

[6] Bird S L, Esterly D M, Perry S G. Off-target deposition of pesticides from agricultural aerial spray applications. Journal of Environmental Quality, 1995; 25(5): 1095-1104.

[7] Huang Y B, Hoffmann W C, Lan Y B, et al. Development of a spray system for an unmanned aerial vehicle platform. Transactions of the ASABE, 2009; 25(6): 803-809.

[8] Huang Y B, Thomson S J. Characterization of spray deposition and drift from a low drift nozzle for aerial application at different application altitudes. Int J Agric \& Biol Eng, 2011; 4(4): 28-33.

[9] Bradley K F, Hoffmann W C, Bagley W E, Hewitt A. Field scale evaluation of spray drift reduction technologies from ground and aerial application systems. Journal of ASTM International, 2011; 8(5): 1-11.

[10] Giles D K, Billing R. Unmanned aerial platforms for spraying: deployment and performance. Aspects Appl. Bio., 2014; 12: 63-69.

[11] Giles D K. Use of Remotely Piloted Aircraft for Pesticide Applications: Issues and Outlook. Outlooks on Pest Management, 2016; 27(5): 213-216.

[12] Giles D K, Billing R, Singh W. Performance results, economic viability and outlook for remotely piloted aircraft for agricultural spraying. Aspects of Applied Biology 132, International Advances in Pesticide Application, 2016; pp.15-21.

[13] Wang C L, He X K, Liu Y J, et al. The small single- and multi-rotor unmanned aircraft vehicles chemical application techniques and control for rice fields in China. Aspects of Applied Biology 132, International Advances in Pesticide Application, 2016; pp.73-81.

[14] He X K, Bonds J, Herbst A, et al. Recent development of unmanned aerial vehicle for plant protection in East Asia. Int J Agric \& Biol Eng, 2017; 10(3): 18-30.

[15] Fritz B K, Kirk I W, Hoffmann W C, et al. Aerial application methods for increasing spray deposition on wheat heads. Applied Engineering in Agriculture, 2006; 22(3): 357-364.

[16] Zhang J, He X K, Song J L, et al. Influence of spraying parameters of unmanned aircraft on droplets deposition. Transactions of the Chinese Society for Agricultural Machinery, 2012; 43(12): 94-96. (in Chinese)

[17] Bae Y, Koo Y M. Flight attitudes and spray patterns of a roll-balanced agricultural unmanned helicopter. Applied Engineering in Agriculture, 2013; 29(5): 675-682.

[18] Qiu B J, Wang L W, Cai D L, et al. Effect of flight altitude and speed of unmanned helicopter on spray deposition uniform. Transactions of the CSAE, 2013; 29(24): 25-32. (in Chinese)

[19] Qin W C, Qiu B J, Xue X Y, et al. Droplet deposition and control effect of insecticides sprayed with an unmanned aerial vehicle against plant hoppers Crop Protection, 2016; 85: 79-88.

[20] Zhang P, Deng L, Lyu Q, et al. Effects of citrus tree-shape and spraying height of small unmanned aerial vehicle on droplet distribution. Int $\mathrm{J}$ Agric \& Biol Eng, 2016; 9(4): 45-52.

[21] Wang C L, Song J L, He X K, et al. Effect of flight parameters on distribution characteristics of pesticide spraying droplets deposition of plant-protection unmanned aerial vehicle. Transactions of the CSAE, 2017; 33(23): 109-116. (in Chinese)

[22] ISO/TC 23/SC 6. ISO 24253-1:2015. Crop protection equipmentSpray deposition test for field crop.

[23] Smith D B. Uniformity and recovery of broadcast spray using fan nozzles. Transactions of the ASAE, 1992; 35: 39-44.

[24] Li J Y, Zhou Z Y, Hu L, et al. Optimization of operation parameters for supplementary pollination in hybrid rice breeding using uniaxial single-rotor electric unmanned helicopter. Transactions of the CSAE, 2014; 30(10): 10-17. (in Chinese)

[25] Shi Q. Numerical simulation for downwash flow field of small-size unmanned helicopter hedgehopping. Journal of Drainage and Irrigation Machinery Engineering (JDIME), 2015; 33(6): 521-525. (in Chinese)

[26] Liao J, Zang Y, Zhou Z Y, et al. Quality evaluation method and optimization of operating parameters in crop aerial spraying technology. Transactions of the CSAE, 2015; 31(Supp.2): 38-46. (in Chinese) 